\title{
A perturbation and generic smoothness of the Vafa-Witten moduli spaces on closed symplectic four-manifolds
}

\author{
Yuuji Tanaka
}

\begin{abstract}
We prove a Freed-Uhlenbeck style generic smoothness theorem for the moduli space of solutions to the Vafa-Witten equations on a closed symplectic four-manifold by using a method developed by Feehan for the study of the $P U(2)$-monopole equations on smooth closed fourmanifolds. We introduce a set of perturbation terms to the VafaWitten equations, and prove that the moduli space of solutions to the perturbed Vafa-Witten equations on a closed symplectic four-manifold for the structure group $S U(2)$ or $S O(3)$ is a smooth manifold of dimension zero for a generic choice of the perturbation parameters.
\end{abstract}

\section{Introduction}

In this article, we consider the Vafa-Witten equations ([VW], [Ma], [Ha], $[\mathrm{W}])$ on a compact symplectic four-manifold. First let us introduce the equations in their original form.

The Vafa-Witten equations. Let $X$ be a closed, oriented, smooth Riemannian four-manifold with Riemannian metric $g$, and let $P \rightarrow X$ be a principal $G$-bundle over $X$ with compact Lie group $G$. We denote by $\mathcal{A}_{P}$ the set of all connections of $P$ and by $\Omega^{+}\left(X, \mathfrak{g}_{P}\right)$ the set of self-dual twoforms valued in the adjoint bundle $\mathfrak{g}_{P}$ of $P$. We consider the following equations for a triple $(A, B, \Gamma) \in \mathcal{A}_{P} \times \Omega^{+}\left(X, \mathfrak{g}_{P}\right) \times \Omega^{0}\left(X, \mathfrak{g}_{P}\right)$ :

$$
\begin{gathered}
d_{A} \Gamma+d_{A}^{*} B=0, \\
F_{A}^{+}+\frac{1}{8}[B \cdot B]+\frac{1}{2}[B, \Gamma]=0,
\end{gathered}
$$

where $[B . B] \in \Omega^{+}\left(X, \mathfrak{g}_{P}\right)$ is defined by a point-wise Lie-algebraic structure on $\Lambda^{+}$together with the bracket of $\mathfrak{g}_{P}$ (see [Ma, $\left.\S \mathrm{A} .1\right]$ or $[\mathrm{Ta}, \S 2]$ for more 
detail). We call these equations the Vafa-Witten equations. The equations (1.1) and (1.2) with a gauge fixing equation form an elliptic system with the index always being zero.

The equations on compact symplectic four-manifolds and a perturbation. We rewrite the equations (1.1) and (1.2) when the underlying manifold $X$ is a compact symplectic four-manifold.

Let $X$ be a compact symplectic four-manifold with symplectic form $\omega$. We take an almost complex structure $J$ compatible with the symplectic form $\omega$. In this setting, the equations (1.1) and (1.2) can be written as follows (see Section 2 for more detail).

$$
\begin{gathered}
\bar{\partial}_{A} \alpha+\bar{\partial}_{A}^{*} \beta=0 \\
F_{A}^{0,2}+\frac{1}{2}[\alpha, \beta]=0, \omega^{2} \wedge\left(i \Lambda F_{A}^{1,1}+\frac{1}{2}\left[\alpha, \alpha^{*}\right]\right)+\left[\beta, \beta^{*}\right]=0
\end{gathered}
$$

where $\Lambda:=(\wedge \omega)^{*}$, and $\alpha \in \Omega^{0,0}\left(X, \mathfrak{g}_{P}\right), \beta \in \Omega^{0,2}\left(X, \mathfrak{g}_{P}\right)$.

We then introduce the following perturbation for the Vafa-Witten equations on a compact symplectic four-manifold:

$$
\begin{gathered}
\bar{\partial}_{A} \alpha+\bar{\partial}_{A}^{*} \beta+\rho(\theta)(\alpha+\beta)=0 \\
F_{A}^{0,2}+\frac{1}{2} \tau_{1}[\alpha, \beta]=0, \omega^{2} \wedge\left(i \Lambda F_{A}^{1,1}+\frac{1}{2} \tau_{2}\left[\alpha, \alpha^{*}\right]\right)+\tau_{3}\left[\beta, \beta^{*}\right]=0
\end{gathered}
$$

where $\rho: T^{*} X \otimes \mathbb{C} \rightarrow \operatorname{Hom}_{\mathbb{C}}\left(\Lambda^{0,0} \oplus \Lambda^{0,2}, \Lambda^{0,1}\right)$ is the Clifford multiplication map, $\tau_{1} \in C^{r}\left(G L\left(\Lambda^{0,2}\right)\right), \tau_{2} \in C^{r}\left(G L\left(\Lambda^{0,0}\right)\right), \tau_{3} \in C^{r}\left(G L\left(\Lambda^{2,2}\right)\right)$ and $\theta \in$ $T^{*} X \otimes \mathbb{C}$ are perturbation parameters. We write $\tau:=\left(\tau_{1}, \tau_{2}, \tau_{3}\right)$, and denote by $\mathcal{P}_{1}$ the Banach space of the perturbation parameters $(\tau, \theta)$, namely, we set $\mathcal{P}_{1}:=C^{r}\left(G L\left(\Lambda^{0,2}\right)\right) \times C^{r}\left(G L\left(\Lambda^{0,0}\right)\right) \times C^{r}\left(G L\left(\Lambda^{2,2}\right)\right) \times C^{r}\left(\Lambda^{1} \otimes \mathbb{C}\right)$.

This perturbation does not depend upon connections. Hence, one needs not be careful about the compatibility with the bubbling-off of connections.

Generic smoothness of the moduli spaces. Before stating results in this article, let us introduce some terminology here first.

Definition 1.1. A connection $A$ of a principal $G$-bundle over $X$ is said to be irreducible if the stabilizer $Z_{A}$ in $\mathcal{G}_{P}$ coincides with the centre of the group $G$, and reducible otherwise.

We also introduce the following notion of rank for sections. 
Definition 1.2. We say a $\mathfrak{g}_{P}$-valued form $\alpha+\beta \in \Gamma\left(\mathfrak{g}_{P} \otimes\left(\Lambda^{0,0} \oplus \Lambda^{0,2}\right)\right)$ is of rank $r$ if, when considered as a section of $\operatorname{Hom}\left(\left(\Lambda^{0,0} \oplus \Lambda^{0,2}\right)^{*}, \mathfrak{g}_{P}\right)$, the section $(\alpha+\beta)(x)$ has rank less than or equal to $r$ at every point $x \in X$ with equality at some point.

We then denote by $\mathcal{M}_{\diamond}^{*}(\tau, \theta)$ the moduli space of solutions $(A,(\alpha, \beta))$ to the perturbed Vafa-Witten equations (1.3) and (1.4) with $A$ irreducible, $\alpha-\bar{\alpha}=0$ and $\alpha+\beta$ being of rank three. We prove the following in Section $3:$

Proposition 1.3. Let $X$ be a closed symplectic four-manifold, and let $P \rightarrow$ $X$ be a principal $G$-bundle over $X$, where we assume that $G$ is either $S U(2)$ or $S O(3)$. Then there is a first category subset $\mathcal{P}_{1}^{\prime} \subset \mathcal{P}_{1}$ such that, for each $(\tau, \theta) \in \mathcal{P}_{1} \backslash \mathcal{P}_{1}^{\prime}$, the moduli space $\mathcal{M}_{\diamond}^{*}(\tau, \theta)$ is a smooth manifold of dimension zero.

Here, a subset of $S^{\prime}$ of a topological space $S$ is said to be a first category subset if $S^{\prime}$ is a countable union of closed subsets of $S$ with empty interior. We mean a generic choice of elements in $S$ by taking an element from $S \backslash S^{\prime}$.

We next consider irreducible solutions to the equations with rank less than or equal to two, and show that there are no such solutions for a generic choice of perturbation parameters. In order to do this we further perturb the equations, that corresponds to moving metrics or almost complex structures of the underlying manifold. More precisely, we introduce an extra perturbation parameter $f \in C^{r}\left(G L\left(T^{*} X\right)\right)$, and consider the following equations:

$$
\begin{gathered}
\bar{\partial}_{A, f} \alpha+\bar{\partial}_{A, f}^{*} \beta+\rho(f(\theta))(\alpha+\beta)=0 \\
P_{f}^{0,2}\left(F_{A}\right)+\frac{1}{2} \tau_{1}[\alpha, \beta]=0, \omega^{2} \wedge\left(i \Lambda P_{f}^{1,1}\left(F_{A}\right)+\frac{1}{2} \tau_{2}\left[\alpha, \alpha^{*}\right]\right)+\tau_{3}\left[\beta, \beta^{*}\right]=0,
\end{gathered}
$$

where $P_{f}^{0,2}$ and $P_{f}^{1,1}$ are the projections to $(0,2)$ and $(1,1)$-parts with respect to the almost complex structure $f^{*} J$, and

$$
\bar{\partial}_{A, f}:=\sum f\left(v^{i}\right) \wedge \nabla_{A, v_{i}}, \quad \bar{\partial}_{A, f}^{*}:=-\sum \iota\left(f\left(v^{i}\right)\right) \nabla_{A, v_{i}},
$$

where $\left\{v^{i}\right\}$ is an orthonormal frame of $\Lambda^{0,1}$, and $\left\{v_{i}\right\}$ is its dual. These $\bar{\partial}_{A, f}$ and $\bar{\partial}_{A, f}^{*}$ can be seen as a variation of the Dirac operator corresponding to moving metrics or almost complex structures of the underlying manifold.

We denote by $\mathcal{P}_{2}:=C^{r}\left(G L\left(T^{*} X\right)\right) \times C^{r}\left(\Lambda^{1} \otimes \mathbb{C}\right)$ the perturbation parameter space and by $\mathcal{M}^{*, 0}(f, \theta)$ the moduli space of solutions $(A,(\alpha, \beta))$ to the equations (1.5) and (1.6) with $A$ irreducible, $\alpha-\bar{\alpha}=0$ and $(\alpha, \beta) \neq 0$. We prove the following in Section 4: 
Proposition 1.4. Let $X$ be a closed symplectic four-manifold, and let $P \rightarrow$ $X$ be a principal $G$-bundle over $X$, where the structure group $G$ is either $S U(2)$ or $S O(3)$. Then there is a first category subset $\mathcal{P}_{2}^{\prime} \subset \mathcal{P}_{2}$ such that for all $(f, \theta) \in \mathcal{P}_{2} \backslash \mathcal{P}_{2}^{\prime}$, the moduli space $\mathcal{M}^{*, 0}(f, \theta)$ contains no solutions $(A,(\alpha, \beta))$ to the perturbed Vafa-Witten equations (1.5) and (1.6) such that $A$ is irreducible, $\alpha-\bar{\alpha}=0$ and $\alpha+\beta$ is of rank one or two.

Our proof of Proposition 1.4 invokes a series of ideas by Feehan [F] in the study of the $P U(2)$-monopole equations, which uses a version of the Sard-Smale theorem (see Section 4.1). Note that Teleman [Te] independently obtained a similar generic-parameter smoothness result for the $P U(2)$-monopole moduli spaces on closed four-manifolds as well.

We now take $\mathcal{P}=C^{r}\left(G L\left(T^{*} X\right)\right) \times C^{r}\left(G L\left(\Lambda^{0,2}\right)\right) \times C^{r}\left(G L\left(\Lambda^{0,0}\right)\right) \times$ $C^{r}\left(G L\left(\Lambda^{2,2}\right)\right) \times C^{r}\left(\Lambda^{1} \otimes \mathbb{C}\right)$ as the perturbation parameter space. Combining Propositions 1.3 and 1.4 above, we obtain the following:

Theorem 1.5. Let $X$ be a closed symplectic four-manifold, and let $P \rightarrow$ $X$ be a principal $G$-bundle over $X$, where the structure group $G$ is either $S U(2)$ or $S O(3)$. We denote by $\mathcal{M}^{*, 0}(f, \tau, \theta)$ the moduli space of solutions $(A,(\alpha, \beta))$ to the perturbed Vafa-Witten equations (1.5) and (1.6) with $A$ irreducible, $\alpha-\bar{\alpha}=0$ and $(\alpha, \beta) \neq 0$. Then there is a first category subset $\mathcal{P}^{\prime} \subset \mathcal{P}$ such that for all $(f, \tau, \theta) \in \mathcal{P} \backslash \mathcal{P}^{\prime}$, the moduli space $\mathcal{M}^{*, 0}(f, \tau, \theta)$ is a smooth manifold of dimension zero.

Note that the $C^{r}$-perturbation parameter space $\mathcal{P}$ and its first category subset in the above theorem can be replaced by $C^{\infty}$-perturbation parameter space and its first category subset by using an argument by Feehan-Leness [FL, §5.1.2].

Acknowledgements. I would like to thank Kael Dixon and Alex Kinsella for helpful conversations to improve the presentation of this article.

\section{Perturbations}

We recall some descriptions of $S p i^{c}$-structures and the Dirac operators on compact symplectic manifolds in Section 2.1. We then describe the perturbations to the equations on compact symplectic four-manifolds in Sections 2.2 and 2.3 . 


\subsection{Spinor bundles and the Dirac operator on symplectic manifolds}

A general reference for $\operatorname{Spin}^{c}$-structures and the Dirac operators is [LM].

Spinor bundles. A spinor bundle $S$ splits into the direct sum of vector bundles $S^{+}$and $S^{-}$, where $S^{+}, S^{-}$are the eigenspaces of the Clifford element of \pm 1 eigenvalues respectively. If $X$ is an oriented smooth four-manifold with Spin $^{c}$-structure, we have the following isomorphism induced from the Clifford multiplication:

$$
T^{*} X \otimes \mathbb{C} \cong \operatorname{Hom}_{\mathbb{C}}\left(S^{+}, S^{-}\right) .
$$

See $[\mathrm{Mo}]$ (or [F, A.3]) for a proof. If $X$ is an almost complex four-manifold, this isomorphism can be written as

$$
T^{*} X \otimes \mathbb{C} \cong \operatorname{Hom}_{\mathbb{C}}\left(\Lambda^{0,0} \oplus \Lambda^{0,2}, \Lambda^{0,1}\right) .
$$

The Dirac operator on symplectic manifolds. Let $E$ be a vector bundle on $X$. The Dirac operator $D_{A}$ associated to a connection $A$ on $E$ is given by the composition:

$$
\Gamma(S) \stackrel{\nabla_{A}}{\longrightarrow} \Gamma\left(T^{*} X \otimes(S \otimes E)\right) \stackrel{\text { metric }}{\longrightarrow} \Gamma(T X \otimes(S \otimes E)) \stackrel{\rho}{\rightarrow} \Gamma(S \otimes E),
$$

where $\rho$ is the Clifford multiplication map.

In the almost complex case, the Dirac operator is written as

$$
D_{A}=\sqrt{2}\left(\bar{\partial}_{A}+\bar{\partial}_{A}^{*}\right)
$$

where $A$ is a connection on $E$. Thus, if the underlying manifold $X$ is a symplectic four-manifold, the Dirac equations become $\bar{\partial}_{A} \alpha+\bar{\partial}_{A}^{*} \beta=0$, where $\alpha \in \Omega^{0,0}(E), \beta \in \Omega^{0,2}(E)$.

\subsection{The equations on symplectic four-manifolds and a per- turbation}

Let $X$ be a compact symplectic four-manifold with symplectic form $\omega$, and let $P$ be a principal $G$-bundle over $X$, where $G$ is a compact Lie group. We take an almost complex structure $J$ compatible with the symplectic form $\omega$.

Let us rewrite the equations (1.1) and (1.2), when the underlying manifold is a compact symplectic four-manifold. This was thoroughly described by Mares $[\mathrm{Ma}, \S 7]$. We follow his notations. First we denote an orthonormal 
frame of $\Lambda^{1}$ by $\left\{e^{0}, e^{1}, e^{2}, e^{3}\right\}$. We write $d z^{1}=e^{0}+i e^{1}, d z^{2}=e^{2}+i e^{3}$. Note that we have $\omega=e^{0} \wedge e^{1}+e^{2} \wedge e^{3}$. We write $B \in \Omega^{+}\left(\mathfrak{g}_{P}\right)$ as $B=B_{1}\left(e^{0} \wedge e^{1}+e^{2} \wedge e^{3}\right)+B_{2}\left(e^{0} \wedge e^{3}+e^{3} \wedge e^{1}\right)+B_{3}\left(e^{0} \wedge e^{3}+e^{1} \wedge e^{2}\right)$. We then define $\alpha \in \Omega^{0,0}\left(X, \mathfrak{g}_{P}\right)$ and $\beta \in \Omega^{0,2}\left(X, \mathfrak{g}_{P}\right)$ by

$$
\alpha:=\Gamma+i B_{1}, \beta:=-\frac{1}{2}\left(B_{2}+i B_{3}\right) d \bar{z}^{1} \wedge d \bar{z}^{2} .
$$

Note that $B$ can be written as $B=B_{1} \omega+\beta+\beta^{*}$. Note also that $\alpha-\bar{\alpha}=0$ if $A$ is irreducible, since $\Gamma=0$ in this case.

With these notations, the equations (1.1) and (1.2) are rewritten as follows.

$$
\begin{gathered}
\bar{\partial}_{A} \alpha+\bar{\partial}_{A}^{*} \beta=0 \\
F_{A}^{0,2}+\frac{1}{2}[\alpha, \beta]=0, \omega^{2} \wedge\left(i \Lambda F_{A}^{1,1}+\frac{1}{2}\left[\alpha, \alpha^{*}\right]\right)+\left[\beta, \beta^{*}\right]=0
\end{gathered}
$$

where $\Lambda:=(\wedge \omega)^{*}$.

Perturbation. We consider the following perturbed Vafa-Witten equations:

$$
\begin{gathered}
\bar{\partial}_{A} \alpha+\bar{\partial}_{A}^{*} \beta+\rho(\theta)(\alpha+\beta)=0 \\
F_{A}^{0,2}+\frac{1}{2} \tau_{1}[\alpha, \beta]=0, \omega^{2} \wedge\left(i \Lambda F_{A}^{1,1}+\frac{1}{2} \tau_{2}\left[\alpha, \alpha^{*}\right]\right)+\tau_{3}\left[\beta, \beta^{*}\right]=0,
\end{gathered}
$$

where $\rho: T^{*} X \otimes \mathbb{C} \rightarrow \operatorname{Hom}_{\mathbb{C}}\left(\Lambda^{0,0} \oplus \Lambda^{0,2}, \Lambda^{0,1}\right)$ is the Clifford multiplication, $\tau_{1} \in C^{r}\left(G L\left(\Lambda^{0,2}\right)\right), \tau_{2} \in C^{r}\left(G L\left(\Lambda^{0,0}\right)\right), \tau_{3} \in C^{r}\left(G L\left(\Lambda^{2,2}\right)\right)$ and $\theta \in T^{*} X \otimes \mathbb{C}$ are perturbation parameters.

Note that this perturbation does not involve connections. In Section 3, we prove that the moduli space of solutions to the above equations (2.3) and (2.4) with $A$ irreducible, $\alpha-\bar{\alpha}=0$ and $\alpha+\beta$ being of rank three is a smooth manifold of dimension zero for a generic choice of the perturbation parameters.

\subsection{Further perturbation}

Following Feehan $[\mathrm{F}, \S 3]$, we consider a perturbation of the Dirac operator. We take $f \in C^{r}\left(G L\left(T^{*} X\right)\right)$, and consider the following:

$$
\bar{\partial}_{A, f}:=\sum f\left(v^{i}\right) \wedge \nabla_{A, v_{i}}, \quad \bar{\partial}_{A, f}^{*}:=-\sum \iota\left(f\left(v^{i}\right)\right) \nabla_{A, v_{i}},
$$


where $\left\{v^{i}\right\}$ is an orthonormal frame of $\Lambda^{0,1}$ and $\left\{v_{i}\right\}$ is its dual. These $\bar{\partial}_{A, f}$

and $\bar{\partial}_{A, f}^{*}$ can be seen as a variation of the Dirac operator corresponding to moving metrics or almost complex structures of the underlying manifold.

We then consider the following equations:

$$
\begin{gathered}
\bar{\partial}_{A, f} \alpha+\bar{\partial}_{A, f}^{*} \beta+\rho(f(\theta))(\alpha+\beta)=0, \\
P_{f}^{0,2}\left(F_{A}\right)+\frac{1}{2} \tau_{1}[\alpha, \beta]=0, \omega^{2} \wedge\left(i \Lambda P_{f}^{1,1}\left(F_{A}\right)+\frac{1}{2} \tau_{2}\left[\alpha, \alpha^{*}\right]\right)+\tau_{3}\left[\beta, \beta^{*}\right]=0,
\end{gathered}
$$

where $\theta \in T^{*} X \otimes \mathbb{C}, P_{f}^{0,2}$ and $P_{f}^{1,1}$ are the projections to $(0,2)$ and $(1,1)$ parts with respect to the almost complex structure $f^{*} J$. We denote the left hand side of $(2.5)$ by $\left(\bar{\partial}_{A,(f, \theta)}+\bar{\partial}_{A,(f, \theta)}^{*}\right)(\alpha+\beta)$.

As in [F, Lem. 3.2], the differential of the above perturbed Dirac operator $\left(\bar{\partial}_{A,(f, \theta)}+\bar{\partial}_{A,(f, \theta)}^{*}\right)$ is given by

$$
\begin{aligned}
& D\left(\bar{\partial}_{A,(f, \theta)}+\bar{\partial}_{A,(f, \theta)}^{*}\right)_{(A,(f, \theta))}(a, \underline{f}, \underline{\theta})(\mathfrak{a}+\mathfrak{b}) \\
&=\sum \underline{f}\left(v^{i}\right) \wedge \nabla_{A, v_{i}} \mathfrak{a}-\sum \iota\left(\underline{f}\left(v^{i}\right)\right) \nabla_{A, v_{i}} \mathfrak{b} \\
&+\rho(f(a))(\mathfrak{a}+\mathfrak{b})+\rho(f(\underline{\theta}))(\mathfrak{a}+\mathfrak{b})
\end{aligned}
$$

where $a \in \Omega^{1}\left(\mathfrak{g}_{P}\right), \underline{f} \in C^{r}\left(\mathfrak{g l}\left(T^{*} X\right)\right), \underline{\theta} \in C^{r}\left(\Lambda^{1} \otimes \mathbb{C}\right)$ and $\mathfrak{a} \in \Omega^{0}\left(\mathfrak{g}_{P}\right), \mathfrak{b} \in$ $\Omega^{0,2}\left(\mathfrak{g}_{P}\right)$.

In Section 4, we prove that there are no rank one or two solutions $(A,(\alpha, \beta))$ to the equations $(2.5)$ and (2.6) with $A$ irreducible, $\alpha-\bar{\alpha}=0$ and $(\alpha, \beta) \neq 0$ for a generic choice of perturbation parameters.

\section{Generic smoothness for the rank three case}

In this section, we prove Proposition 1.3. In order to do that we consider the parametrized moduli space, and prove that it is a smooth manifold (Proposition 3.1). Then Proposition 1.3 follows from Proposition 3.1.

\subsection{Parametrized moduli space}

Let $X$ be a compact symplectic four-manifold with symplectic form $\omega$, and let $P$ be a principal $G$-bundle over $X$. From now on, $G$ is either $S U(2)$ or $S O(3)$. We take an almost complex structure $J$ compatible with the symplectic form $\omega$. 
We denote by $\mathcal{A}_{k}^{2}(P)$ the $L_{k}^{2}$-completion of the space of connections on $P$, and by $\mathcal{G}(P)=\mathcal{G}_{k+1}^{2}(P)$ the $L_{k+1}^{2}$-completion of the gauge group. We set

$$
\mathcal{C}(P):=\mathcal{A}_{k}^{2}(P) \times L_{k}^{2}\left(\mathfrak{g}_{P} \otimes\left(\Lambda^{0,0} \oplus \Lambda^{0,2}\right)\right),
$$

and $\mathcal{P}_{1}:=C^{r}\left(G L\left(\Lambda^{0,2}\right)\right) \times C^{r}\left(G L\left(\Lambda^{0,0}\right)\right) \times C^{r}\left(G L\left(\Lambda^{2,2}\right)\right) \times C^{r}\left(\Lambda^{1} \otimes \mathbb{C}\right)$. This $\mathcal{P}_{1}$ is the parameter space for the perturbation described in Section 2.2. We denote the quotient $\mathcal{C}(P) / \mathcal{G}(P)$ by $\mathcal{B}(P)$.

We define

$$
s: \mathcal{C}(P) \times \mathcal{P}_{1} \rightarrow L_{k-1}^{2}\left(\mathfrak{g}_{P} \otimes \Lambda^{0,1}\right) \times L_{k-1}^{2}\left(\mathfrak{g}_{P} \otimes\left(\Lambda^{0,2} \oplus \Lambda^{1,1}\right)\right)
$$

by $s(A,(\alpha, \beta), \tau, \theta):=\left(s_{1}(A,(\alpha, \beta), \tau, \theta), s_{2}(A,(\alpha, \beta), \tau, \theta)\right)$, where

$$
\begin{aligned}
s_{1}(A,(\alpha, \beta), \tau, \theta) & :=\bar{\partial}_{A} \alpha+\bar{\partial}_{A}^{*} \beta+\rho(\theta)(\alpha+\beta), \\
s_{2}(A,(\alpha, \beta), \tau, \theta) & :=F_{A}^{0,2}+\frac{1}{2} \tau_{1}[\alpha, \beta]+\Lambda F_{A}^{1,1} \wedge \omega \\
& +\frac{1}{2} \tau_{2}\left[\alpha, \alpha^{*}\right] \wedge \omega+\Lambda \tau_{3}\left[\beta, \beta^{*}\right] .
\end{aligned}
$$

This is a $\mathcal{G}(P)$-equivariant map, where the action of $\mathcal{G}(P)$ on $\mathcal{P}_{1}$ is taken to be trivial. Here $\rho: T^{*} X \otimes \mathbb{C} \rightarrow \operatorname{Hom}_{\mathbb{C}}\left(\Lambda^{0,0} \otimes \Lambda^{0,2}, \Lambda^{0,1}\right)$ is the Clifford multiplication map, and $\tau:=\left(\tau_{1}, \tau_{2}, \tau_{3}\right) \in \mathcal{P}_{1}$. We say $M(P):=s^{-1}(0) / \mathcal{G}(P) \subset$ $\mathcal{B}(P) \times \mathcal{P}_{1}$ the parametrized moduli space.

We denote by $\mathcal{B}_{\diamond}^{*}(P)$ gauge equivalence classes of pairs $(A,(\alpha, \beta)) \in \mathcal{C}(P)$ with $A$ irreducible, $\alpha-\bar{\alpha}=0$ and $\alpha+\beta$ being of rank three. We set $M_{\diamond}^{*}(P):=M(P) \cap\left(\mathcal{B}_{\diamond}^{*}(P) \times \mathcal{P}_{1}\right)$. We then have the following:

Proposition 3.1. The zero set $s^{-1}(0)$ in $\mathcal{B}_{\diamond}^{*}(P) \times \mathcal{P}_{1}$ is regular, in particular, the parametrized moduli space $M_{\diamond}^{*}(P)$ is a smooth Banach submanifold of $\mathcal{B}_{\diamond}^{*}(P) \times \mathcal{P}_{1}$.

We prove Proposition 3.1 in Section 3.2. Proposition 1.3 follows from Proposition 3.1 as described below.

Proof of Proposition 1.3. Note that $s$ is a Fredholm section if it is restricted to $\mathcal{B}(P) \times\{(\tau, \theta)\}$ for a perturbation parameter $(\tau, \theta)$. Thus, by the SardSmale theorem ([DK, Prop 4.3.11]), there exists a first category subset $\mathcal{P}_{1}^{\prime}$ such that the zero set of $s$ in $\mathcal{B}_{\diamond}^{*}(P)$ is regular for $(\tau, \theta) \in \mathcal{P}_{1} \backslash \mathcal{P}_{1}^{\prime}$. Hence, $\mathcal{M}_{\diamond}^{*}(\tau, \theta)=s^{-1}(0) \cap \mathcal{B}_{\diamond}^{*}(P)$ is a smooth manifold for generic $C^{r}$-parameters $(\tau, \theta)$. 


\subsection{Proof of Proposition 3.1}

In this section, we prove Proposition 3.1. We follow an argument by Feehan [F, $\S 2.2]$ (see also [DK, §4.3.5]). First we consider the linearisation $D s=$ $\left(D s_{1}, D s_{2}\right): L_{k}^{2}\left(\mathfrak{g}_{P} \otimes \Lambda^{0,1}\right) \times L_{k}^{2}\left(\mathfrak{g}_{P} \otimes\left(\Lambda^{0,0} \oplus \Lambda^{0,2}\right)\right) \times \mathcal{P}_{1} \rightarrow L_{k-1}^{2}\left(\mathfrak{g}_{P} \otimes \Lambda^{0,1}\right) \times$ $L_{k-1}^{2}\left(\mathfrak{g}_{P} \otimes\left(\Lambda^{1,1} \oplus \Lambda^{0,2}\right)\right)$ of $s$ at $(A,(\alpha, \beta), \tau, \theta) \in s^{-1}(0)$, where

$$
\begin{aligned}
D s_{1}((\underline{\tau}, \underline{\theta}), a,(\mathfrak{a}, \mathfrak{b}))=\bar{\partial}_{A} \mathfrak{a}+\bar{\partial}_{A}^{*} \mathfrak{b}+\rho(\theta)(\mathfrak{a}+\mathfrak{b})+\rho(\underline{\theta})(\alpha+\beta) \\
D s_{2}((\underline{\tau}, \underline{\theta}), a,(\mathfrak{a}, \mathfrak{b}))=\bar{\partial}_{A} a+\partial_{A} a+\frac{1}{2} \tau_{1}([\mathfrak{a}, \beta]+[\alpha, \mathfrak{b}])+\frac{1}{2} \tau_{1} \tau_{1}[\alpha, \beta] \\
+\frac{1}{2} \tau_{2}\left(\left[\alpha, \mathfrak{a}^{*}\right]+\left[\mathfrak{a}, \alpha^{*}\right]\right) \wedge \omega+\frac{1}{2} \underline{\tau_{2}} \tau_{2}\left(\left[\alpha, \alpha^{*}\right]\right) \wedge \omega \\
+\Lambda \tau_{3}\left(\left[\beta, \mathfrak{b}^{*}\right]+\left[\mathfrak{b}, \beta^{*}\right]\right)+\Lambda \underline{\tau_{3}} \tau_{3}\left(\left[\beta, \beta^{*}\right]\right) .
\end{aligned}
$$

We then suppose for a contradiction that there exists $(\delta, v) \in C^{0}\left(\mathfrak{g}_{P} \otimes\right.$ $\left.\Lambda^{0,1}\right) \times C^{0}\left(\mathfrak{g}_{P} \otimes\left(\Lambda^{1,1} \oplus \Lambda^{0,2}\right)\right)$ with $(\delta, v) \neq 0$ such that

$$
\left\langle D s_{1}(a,(\mathfrak{a}, \mathfrak{b}), \underline{\tau}, \underline{\theta}), \delta\right\rangle_{L^{2}}=0, \quad\left\langle D s_{2}(a,(\mathfrak{a}, \mathfrak{b}), \underline{\tau}, \underline{\theta}), v\right\rangle_{L^{2}}=0 .
$$

By setting $(\mathfrak{a}, \mathfrak{b})=0$ in the first equation of (3.1), we get

$$
\langle\rho(\underline{\theta})(\alpha+\beta), \delta\rangle_{L^{2}}=0
$$

for $\underline{\theta} \in C^{r}\left(\Lambda^{1} \otimes \mathbb{C}\right)$.

Lemma 3.2. Assume that $\alpha+\beta \in C^{0}\left(\mathfrak{g}_{P} \otimes\left(\Lambda^{0,0} \oplus \Lambda^{0,2}\right)\right)$ and $\delta \in C^{0}\left(\mathfrak{g}_{P} \otimes\right.$ $\left.\Lambda^{0,1}\right)$ satisfy (3.2). Then $\alpha+\beta$ and $\delta$ have orthogonal images in $\mathfrak{g}_{P}$ at each point of $X$, in particular,

$$
\operatorname{rank}_{\mathbb{R}}(\alpha+\beta)(x)+\operatorname{rank}_{\mathbb{R}} \delta(x) \leq 3
$$

at each point $x \in X$.

proof. In (3.2), $\underline{\theta} \in C^{r}\left(\Lambda^{1} \otimes \mathbb{C}\right)$ is arbitrary, thus, we get the point-wise identity

$$
\left\langle\rho\left(\underline{\theta}_{x}\right)(\alpha+\beta)(x), \delta(x)\right\rangle_{x}=0
$$

for all $\underline{\theta}_{x} \in\left(T^{*} X\right)_{x} \otimes \mathbb{C}$.

We then recall the following.

Lemma 3.3 ([F], Lem. 2.3). Let $U$ and $V$ be complex vector spaces with $\operatorname{dim} U \leq \operatorname{dim} V$, and let $W$ be a real vector space. We take $M \in U^{*} \otimes_{\mathbb{R}} W$ and $N \in V^{*} \otimes_{\mathbb{R}} W$. Then, if $\langle M P, N\rangle_{V^{*} \otimes_{\mathbb{R}} W}=0$ for all $P \in \operatorname{Hom}_{\mathbb{C}}(V, U)$, we get $\operatorname{Ran} M \perp \operatorname{Ran} N$ in $W$, in particular, $\operatorname{rank}_{\mathbb{R}} M+\operatorname{rank}_{\mathbb{R}} N \leq \operatorname{dim}_{\mathbb{R}} W$. 
Since $\rho$ gives a complex linear isomorphism

$$
\left(T^{*} X\right)_{x} \otimes_{\mathbb{R}} \mathbb{C} \rightarrow \operatorname{Hom}_{\mathbb{C}}\left(\Lambda^{0,1} \oplus \Lambda^{0,2}, \Lambda^{0,1}\right)_{x},
$$

we can invoke Lemma 3.3 to obtain the assertion.

As $(A,(\alpha, \beta)) \in \mathcal{C}_{\diamond}^{*}(P)$ and $\alpha+\beta$ is $C^{r}$ for some $r$, there is a non-empty open subset $U \subset X$ on which $\operatorname{rank}_{\mathbb{R}}(\alpha+\beta)(x)=3$ for all $x \in U$. Then Lemma 3.2 implies that $\operatorname{rank} \delta(x)=0$ for all $x \in U$, namely, $\delta \equiv 0$ on $U$.

In a similar way, by setting $(a,(\mathfrak{a}, \mathfrak{b}))=0$ in the second equation of $(3.1)$, we get

$$
\left.\left\langle\frac{1}{2} \underline{\tau_{1}} \tau_{1}[\alpha, \beta]+\frac{1}{2} \underline{\tau_{2}} \tau_{2}\left[\alpha, \alpha^{*}\right] \wedge \omega+\Lambda \underline{\tau_{3}} \tau_{3}\left[\beta, \beta^{*}\right]\right), v\right\rangle_{L^{2}(X)}=0
$$

for all $\underline{\tau_{1}} \in C^{r}\left(\mathfrak{g l}\left(\Lambda^{0,2}\right)\right), \underline{\tau_{2}} \in C^{r}\left(\mathfrak{g l}\left(\Lambda^{0,0}\right)\right)$ and $\underline{\tau_{3}} \in C^{r}\left(\mathfrak{g l}\left(\Lambda^{2,2}\right)\right)$.

Lemma 3.4. If $v \in C^{0}\left(\mathfrak{g}_{P} \otimes\left(\Lambda^{1,1} \oplus \Lambda^{0,2}\right)\right)$ and $\alpha+\beta \in C^{0}\left(\mathfrak{g}_{P} \otimes\left(\Lambda^{0,0} \oplus \Lambda^{0,2}\right)\right)$ satisfy (3.3), then $v$ and $\frac{1}{2} \tau_{1}[\alpha, \beta]+\frac{1}{2} \tau_{2}\left[\alpha, \alpha^{*}\right] \wedge \omega+\Lambda \tau_{3}\left[\beta, \beta^{*}\right] \in \operatorname{Hom}\left(\left(\Lambda^{1,1} \oplus\right.\right.$ $\left.\left.\Lambda^{0,2}\right)^{*}, \mathfrak{g}_{P}\right)$ have orthogonal images in $\mathfrak{g}_{P}$ at each point in $X$, in particular,

$$
\operatorname{rank}_{\mathbb{R}} v(x)+\operatorname{rank}_{\mathbb{R}}\left(\frac{1}{2}[\alpha, \beta]+\frac{1}{2}\left[\alpha, \alpha^{*}\right] \wedge \omega+\Lambda\left[\beta, \beta^{*}\right]\right)(x) \leq 3
$$

at each $x \in X$.

proof. As $\underline{\tau_{1}} \in C^{r}\left(\mathfrak{g l}\left(\Lambda^{0,2}\right)\right), \underline{\tau_{2}} \in C^{r}\left(\mathfrak{g l}\left(\Lambda^{0,0}\right)\right)$ and $\underline{\tau_{3}} \in C^{r}\left(\mathfrak{g l}\left(\Lambda^{2,2}\right)\right)$ are arbitrary, we get the following point-wise identity:

$$
\left\langle\left(\frac{1}{2} \underline{\tau_{1}} \tau_{1}[\alpha, \beta]+\frac{1}{2} \underline{\tau_{2}} \tau_{2}\left[\alpha, \alpha^{*}\right] \wedge \omega+\Lambda \underline{\tau_{3}} \tau_{3}\left[\beta, \beta^{*}\right]\right)(x), v(x)\right\rangle_{x}=0
$$

for all $\underline{\tau_{1}}(x) \in C^{r}\left(\mathfrak{g l}\left(\left.\Lambda^{0,2}\right|_{x}\right)\right), \underline{\tau_{2}}(x) \in C^{r}\left(\mathfrak{g l}\left(\left.\Lambda^{0,0}\right|_{x}\right)\right), \underline{\tau_{3}}(x) \in C^{r}\left(\mathfrak{g l}\left(\left.\Lambda^{2,2}\right|_{x}\right)\right)$ and for all $x \in X$. Then we again invoke Lemma 3.3 to obtain the assertion.

The following is due to Mares [Ma, §4.1.1].

Lemma 3.5 ([Ma]). Let $(A, \alpha+\beta)$ be an irreducible solution to the equation, and let $x \in X$. Then $\operatorname{rank}_{\mathbb{R}}\left(\frac{1}{2}[\alpha, \beta]+\frac{1}{2}\left[\alpha, \alpha^{*}\right] \wedge \omega+\Lambda\left[\beta, \beta^{*}\right]\right)(x)=3$ if and only if $\operatorname{rank}_{\mathbb{R}}(\alpha+\beta)(x)=3$.

From Lemma 3.5, if rank $(\alpha+\beta)(x)=3$ for all $x \in U$, then $\operatorname{rank}_{\mathbb{R}} \frac{1}{2}[\alpha, \beta]+$ $\left.\frac{1}{2}\left[\alpha, \alpha^{*}\right] \wedge \omega+\Lambda\left[\beta, \beta^{*}\right]\right)(x)=3$ for all $x \in U$. Thus Lemma 3.4 implies $\operatorname{rank} v(x)=0$ for all $x \in U$. Therefore, $v \equiv 0$ on $U$. Hence $(\delta, v) \equiv 0$ on $U$. Thus by unique continuation for the Laplacian $(D s)(D s)^{*}$ implies that $(\delta, v) \equiv 0$ on the whole of $X$. This is a contradiction. 


\section{Non-existence of rank one and two cases}

In this section, we prove Proposition 1.4. Except modifications stated as Proposition 4.2 in Section 4.2 and Proposition 4.4 in Section 4.3, the proof goes in a similar way to the case for the $P U(2)$-monopole equations by Feehan $[\mathrm{F}]$. In Section 4.1, we introduce some terminology and a version of the Sard-Smale theorem from $[\mathrm{F}]$, which we use in the later sections. We give a characterization of the rank one and two sections in Section 4.2. In Section 4.3, we prove a surjectivity of some linear operator. We then prove Proposition 1.4 in Section 4.4 by using the Sard-Smale theorem.

\subsection{Banach spaces, Fredholm operators and the Sard-Smale theorem}

Let $V$ be a Banach space. For each $k \geq 1$, we define the infinite dimensional Grassmannian by

$$
\mathbb{G}_{k}(V):=\{K \subset V: K \text { is a } k \text {-dimensional subspace of } V\} \text {. }
$$

We write $\mathbb{P}(V)=\mathbb{G}_{1}(V)$. We also define the infinite dimensional flag manifold by

$$
\mathbb{F}_{k}(V):=\left\{(\ell, K) \in \mathbb{P}(V) \times \mathbb{G}_{k}(V): \ell \subset K\right\} .
$$

We denote the projections by $\pi_{1}: \mathbb{F}_{k}(V) \rightarrow \mathbb{P}(V)$ and $\pi_{2}: \mathbb{F}_{k}(V) \rightarrow \mathbb{G}_{k}(V)$. Note that both $\pi_{1}$ and $\pi_{2}$ are submersions (see Claims 4.2 and 4.3 in [F]).

We next consider a smooth submanifold $Z \in \mathbb{P}(V)$. We set $I_{k}(Z):=$ $\pi_{2}\left(\pi_{1}^{-1}(Z)\right) \subset \mathbb{G}_{k}(V)$. As $\pi_{1}$ is a submersion, $\tilde{I}_{k}(Z):=\pi_{1}^{-1}(Z) \subset \mathbb{F}_{k}(V)$ is a smooth submanifold. Note that, however, $I_{k}(Z)$ is not necessarily a submanifold.

Space of Fredholm operators. Let $V_{1}, V_{2}$ be Banach spaces. We denote by $\operatorname{Fred}_{n}\left(V_{1}, V_{2}\right)$ the space of bounded Fredholm operators of index $n$ in the Banach space of the bounded operators. In our case, we take $V_{1}:=$ $L_{k}^{2}\left(\mathfrak{g}_{P} \otimes\left(\Lambda_{I}^{0,0} \oplus \Lambda^{0,2}\right)\right)$, where $\Lambda_{I}^{0,0}:=\left\{\alpha \in \Lambda^{0,0}: \alpha-\bar{\alpha}=0\right\}$ and $V_{2}:=$ $L_{k-1}^{2}\left(\mathfrak{g}_{P} \otimes \Lambda^{0,1}\right)$ in the subsequent sections. We define

$$
\text { Fred }_{k, n}:=\left\{A \in \operatorname{Fred}_{n}\left(V_{1}, V_{2}\right): \operatorname{dim}_{\mathbb{R}} \operatorname{ker} A=k\right\} .
$$

We also define a map

$$
\pi: \operatorname{Fred}_{k, n}\left(V_{1}, V_{2}\right) \rightarrow \mathbb{G}_{k}\left(V_{1}\right)
$$


by $A \mapsto \operatorname{ker} A$. This is smooth, and a submersion ([F, Lem. 4.5]). We then define the following flag manifold for each $\operatorname{Fred}_{k, n}\left(V_{1}, V_{2}\right)$ :

$$
\operatorname{Flag}_{k, n}\left(V_{1}, V_{2}\right):=\left\{(\ell, A) \in \mathbb{P}\left(V_{1}\right) \times \operatorname{Fred}_{k, n}\left(V_{1}, V_{2}\right): \ell \in \operatorname{ker} A\right\} .
$$

This Flag $\operatorname{lag}_{k, n}\left(V_{1}, V_{2}\right)$ is a smooth submanifold of $\mathbb{P}\left(V_{1}\right) \times \operatorname{Fred}_{k, n}\left(V_{1}, V_{2}\right)$ and the canonical map $\varpi: \operatorname{Flag}_{k, n}\left(V_{1}, V_{2}\right) \rightarrow \mathbb{F}_{k}\left(V_{1}\right)$ is a submersion (see $[\mathrm{F}$, Lem. 4.6]).

The Sard-Smale theorem. We state a version of the Sard-Smale theorem from $[\mathrm{F}]$.

Proposition 4.1 ([F], Prop.4.12). Let $\mathcal{C}, \mathcal{P}, \mathcal{F}$ be $C^{\infty}$-Banach manifolds. Suppose that $M \subset \mathcal{C} \times \mathcal{P}$ is a $C^{\infty}$-Banach submanifold, and the restriction $\pi_{M, \mathcal{P}}: M \rightarrow \mathcal{P}$ of the projection map $\pi_{\mathcal{P}}: \mathcal{C} \times \mathcal{P} \rightarrow \mathcal{P}$ is Fredholm. Let $\underline{v}: M \subset \mathcal{C} \times \mathcal{P} \rightarrow \mathcal{F}$ be a $C^{\infty}$-map which is transverse to a $C^{\infty}$-Banach submanifold $\mathcal{J} \subset \mathcal{F}$. Then there exists a first category subset $\mathcal{P}^{\prime} \subset \mathcal{P}$ such that the following holds. For all $p \in \mathcal{P} \backslash \mathcal{P}^{\prime}$,

- $M:=\pi_{M, \mathcal{P}}^{-1}(p)$ is a $C^{\infty}$-manifold of dimension ind $\left(\pi_{M, \mathcal{P}}\right)_{p}<\infty$;

- $v:=\underline{v}(\cdot, p): M \rightarrow \mathcal{F}$ is transverse to the submanifold $\mathcal{J} \subset \mathcal{F}$; and

- $Z:=v^{-1}(\mathcal{J}) \subset M$ is a $C^{\infty}$-submanifold of codimension $\operatorname{codim}(Z, M)=$ $\operatorname{codim}(\mathcal{J}, \mathcal{F})$.

We use this to prove Proposition 1.4 in Section 4.4.

\subsection{Rank one and two loci}

We take $k \geq 4$ so that $V_{1}=L_{k-1}^{2}\left(\mathfrak{g}_{P} \otimes\left(\Lambda_{I}^{0,0} \oplus \Lambda^{0,2}\right)\right) \subset C^{0}\left(\mathfrak{g}_{P} \otimes\left(\Lambda_{I}^{0,0} \oplus \Lambda^{0,2}\right)\right)$.

We think of $C^{0}\left(\mathfrak{g}_{P} \otimes\left(\Lambda_{I}^{0,0} \oplus \Lambda^{0,2}\right)\right)$ as $C^{0}\left(\operatorname{Hom}_{\mathbb{R}}\left(\left(\Lambda_{I}^{0,0} \oplus \Lambda^{0,2}\right)^{*}, \mathfrak{g}_{P}\right)\right)$, and define a determinant map

$$
h: C^{0}\left(\mathfrak{g}_{P} \otimes\left(\Lambda_{I}^{0,0} \oplus \Lambda^{0,2}\right)\right) \rightarrow C^{0}\left(\operatorname{det}\left(\Lambda_{I}^{0,0} \oplus \Lambda^{0,2}\right) \otimes \operatorname{det}\left(\mathfrak{g}_{P}\right)\right)
$$

by $\varphi \in C^{0}\left(\mathfrak{g}_{P} \otimes\left(\Lambda_{I}^{0,0} \oplus \Lambda^{0,2}\right)\right) \mapsto \operatorname{det} \varphi$, where $\operatorname{det}\left(\Lambda_{I}^{0,0} \oplus \Lambda^{0,2}\right)=\Lambda^{3}\left(\Lambda_{I}^{0,0} \oplus\right.$ $\left.\Lambda^{0,2}\right)$ and $\operatorname{det}\left(\mathfrak{g}_{P}\right)=\Lambda^{3} \mathfrak{g}_{P}$. Then $\varphi \in V_{1}$ with $\varphi \neq 0$ is of rank one or two if and only if $h(\varphi)=0$. We define

$$
\mathcal{Z}:=\left\{[\varphi] \in \mathbb{P}\left(V_{1}\right): h(\varphi)=0\right\}
$$


where $[\varphi]$ is the line $\mathbb{R} \cdot \varphi \subset V_{1}$. We denote by $\mathcal{Z}^{\prime}$ the smooth part of $\mathcal{Z}$.

As in the case of the $P U(2)$-monopole equations [F, Lem 4.7], one obtains the following:

Proposition 4.2. Let $[\varphi] \in \mathcal{Z}$. We assume that $\{\varphi \neq 0\}$ is a dense open subset of $X$. Then the determinant map $h: C^{0}\left(\mathfrak{g}_{P} \otimes\left(\Lambda_{I}^{0,0} \oplus \Lambda^{0,2}\right)\right) \rightarrow$ $C^{0}\left(\operatorname{det}\left(\Lambda_{I}^{0,0} \oplus \Lambda^{0,2}\right) \otimes \operatorname{det}\left(\mathfrak{g}_{P}\right)\right)$ vanishes transversely at $\varphi$, and $[\varphi]$ is a smooth point of $\mathcal{Z}$. In addition, the tangent space $T_{[\varphi]} \mathcal{Z}$ has both infinite dimension and infinite codimension in $T_{[\varphi]} \mathbb{P}\left(V_{1}\right)$, in particular, we have $\operatorname{codim}\left(\mathcal{Z}^{\prime}, \mathbb{P}\left(V_{1}\right)\right)=\infty$.

proof. We take a local orthonormal frame $\left\{\phi_{1}, \phi_{2}, \phi_{3}\right\}$ for $\mathfrak{g}_{P}$, and local orthonormal frame $\left\{e_{1}, e_{2}, e_{3}\right\}$ for $\Lambda_{I}^{0,0} \oplus \Lambda^{0,2}$ on an open subset $U \subset X$ so that $\varphi=\left(\begin{array}{lll}\varphi_{11} & \varphi_{12} & \varphi_{13} \\ \varphi_{21} & \varphi_{22} & \varphi_{23} \\ \varphi_{31} & \varphi_{32} & \varphi_{33}\end{array}\right)$. Then the differential of $h$ at $\varphi$ with respect to these frame is given by

$(D h)_{\varphi}(\varphi)=$

$\sum_{\sigma \in \mathfrak{S}_{3}}\left\{\operatorname{sgn}(\sigma)\left(\underline{\varphi_{1 \sigma(1)}} \varphi_{2 \sigma(2)} \varphi_{3 \sigma(3)}+\varphi_{1 \sigma(1)} \underline{\varphi_{2 \sigma(2)}} \varphi_{3 \sigma(3)}+\varphi_{1 \sigma(1)} \varphi_{2 \sigma(2)} \underline{\varphi_{3 \sigma(3)}}\right)\right\}$,

where $\underline{\varphi}=\left(\begin{array}{lll}\frac{\varphi_{11}}{\varphi_{21}} & \frac{\varphi_{12}}{\varphi_{22}} & \frac{\varphi_{13}}{\varphi_{31}} \\ \frac{\varphi_{32}}{\varphi_{33}} & \underline{\varphi_{33}}\end{array}\right) \in C^{\infty}(U, \mathfrak{g l}(3, \mathbb{R}))$.

We now suppose for a contradiction that there exists $\psi \in \operatorname{coker}(D h)_{\varphi}$ so that $\left\langle(D h)_{\varphi}(\underline{\varphi}), \psi\right\rangle_{L^{2}}=0$ for all $\underline{\varphi} \in C^{0}\left(V_{1}\right)$. From the assumption, $\{\varphi \neq 0\}$ is dense in $U$, so the union of the complements of each zero set of $\varphi_{i j}^{\prime} s$ is a dense open subset of $U$, hence we get $\psi \equiv 0$ on $U$. Since $U$ was arbitrary, $\psi \equiv 0$ on $X$. This is a contradiction.

We denote by $M^{*, 0}(P)$ the parametrized moduli space for the perturbed Vafa-Witten equations (2.5) and (2.6) with $A$ irreducible, $\alpha-\bar{\alpha}=0$ and $(\alpha, \beta) \neq 0$. From Proposition 4.2, we get the following:

Corollary 4.3. If $(A, \varphi=(\alpha, \beta), \tau, \theta)$ is in $M^{*, 0}(P)$ so that $h(\varphi)=0$, then $[\varphi]$ is a smooth point of $\mathcal{Z} \subset \mathbb{P}\left(V_{1}\right)$, that is, $\pi\left(M^{*, 0}(P)\right) \subset \mathcal{Z}^{\prime}$, where $\pi: M^{*, 0}(P) \rightarrow \mathbb{P}\left(V_{1}\right)$ is the projection.

For each $k \geq n$, we now define

$$
\tilde{I}_{k}(\mathcal{Z}):=\pi_{1}^{-1}(\mathcal{Z}) \subset \mathbb{F}_{k}\left(V_{1}\right),
$$


and $I_{k}(\mathcal{Z}):=\pi_{2}\left(\tilde{I}_{k}(\mathcal{Z})\right) \subset \mathbb{G}_{k}\left(V_{1}\right)$. By Corollary 4.3, we only consider $I_{k}\left(\mathcal{Z}^{\prime}\right)$ and $\tilde{I}_{k}\left(\mathcal{Z}^{\prime}\right)$ for our purpose. As $\pi_{1}: \mathbb{F}_{k}(V) \rightarrow \mathbb{P}(V)$ is a submersion ([F, Claim 4.2]), $\tilde{I}_{k}\left(\mathcal{Z}^{\prime}\right)$ is a smooth submanifold of $\mathbb{F}_{k}\left(V_{1}\right)$ with codimension

$$
\operatorname{codim}\left(\tilde{I}_{k}\left(\mathcal{Z}^{\prime}\right), \mathbb{F}_{k}\left(V_{1}\right)\right)=\operatorname{codim}\left(\mathcal{Z}^{\prime}, \mathbb{P}\left(V_{1}\right)\right)=\infty .
$$

We put $J_{k}\left(\mathcal{Z}^{\prime}\right):=\pi^{-1}\left(I_{k}\left(\mathcal{Z}^{\prime}\right)\right) \subset \operatorname{Fred}_{k, n}\left(V_{1}, V_{2}\right)$, where $\pi: \operatorname{Fred}_{k, n}\left(V_{1}, V_{2}\right) \rightarrow$ $\mathbb{G}_{k}\left(V_{1}\right)$. We now define the rank one and two loci $\tilde{J}_{k}\left(\mathcal{Z}^{\prime}\right):=\varpi^{-1}\left(\tilde{I}_{k}\left(\mathcal{Z}^{\prime}\right)\right) \subset$ $\operatorname{Flag}_{k, n}\left(V_{1}, V_{2}\right)$, where $\varpi: \operatorname{Flag}_{k, n}\left(V_{1}, V_{2}\right) \rightarrow \mathbb{F}_{k}\left(V_{1}\right)$ is the canonical map. As $\varpi: \operatorname{Flag}_{k, n}\left(V_{1}, V_{2}\right) \rightarrow \mathbb{F}_{k}\left(V_{1}\right)$ is a submersion ([F, Lem 4.6]), the rank one and two loci $\tilde{J}_{k}\left(\mathcal{Z}^{\prime}\right)$ is a smooth submanifold, and we get

$$
\operatorname{codim}\left(\tilde{J}_{k}\left(\mathcal{Z}^{\prime}\right), \operatorname{Flag}_{k, n}\left(V_{1}, V_{2}\right)\right)=\operatorname{codim}\left(\tilde{I}_{k}\left(\mathcal{Z}^{\prime}\right), \mathbb{F}_{k}\left(V_{1}\right)\right)=\infty .
$$

\subsection{A surjectivity}

In this section and the upcoming one, we take $\mathcal{P}_{2}:=C^{r}\left(G L\left(T^{*} X\right)\right) \times C^{r}\left(\Lambda^{1} \otimes\right.$ $\mathbb{C})$ as the perturbation parameter space, since the perturbation parameter $\tau=\left(\tau_{1}, \tau_{2}, \tau_{3}\right)$ is not needed in the proof of Proposition 1.4.

We denote by $\mathcal{C}^{*}(P)$ the set of pairs $(A,(\alpha, \beta)) \in \mathcal{C}(P)$ with $A$ irreducible and $\alpha-\bar{\alpha}=0$. As in $[\mathrm{F}, \S 4.4]$ (see also [DK, $\S 4.3 .3]$ ), we consider the period map

$$
v: \mathcal{C}^{*}(P) \times \mathcal{P}_{2} \rightarrow \operatorname{Fred}_{n}\left(V_{1}, V_{2}\right),
$$

defined by $(A,(\alpha, \beta), f, \theta) \mapsto\left(\bar{\partial}_{A,(f, \theta)}+\bar{\partial}_{A,(f, \theta)}^{*}\right)$. The differential of $v$ at $(A,(\alpha, \beta), f, \theta)$

$$
(D v)_{(A,(\alpha, \beta), f, \theta)}: T_{(A,(\alpha, \beta))} \mathcal{C}^{*}(P) \oplus T_{(f, \theta)} \mathcal{P}_{2} \rightarrow \operatorname{Hom}_{\mathbb{R}}\left(V_{1}, V_{2}\right)
$$

is given by $(a,(\mathfrak{a}, \mathfrak{b}), \underline{f}, \underline{\theta}) \mapsto D\left(\bar{\partial}_{A,(f, \theta)}+\bar{\partial}_{A,(f, \theta)}^{*}\right)_{(A,(f, \theta))}(a, \underline{f}, \underline{\theta})$.

We denote by $\mathcal{C}^{*, 0}(P)$ the set of pairs $(A,(\alpha, \beta)) \in \mathcal{C}(P)$ with $A$ irreducible, $\alpha-\bar{\alpha}=0$ and $(\alpha, \beta) \neq 0$, and by $\mathcal{B}^{*, 0}(P)$ the quotient $\mathcal{C}^{*, 0}(P) / \mathcal{G}(P)$. We set $M^{*, 0}(P)=M(P) \cap\left(\mathcal{B}^{*, 0}(P) \times \mathcal{P}_{2}\right)$, where $M(P)$ is the parametrized moduli space for the equations (2.5) and (2.6). In this section, we prove the following:

Proposition 4.4. Let $(A,(\alpha, \beta), f, \theta) \in M^{*, 0}(P)$. Then, the following is surjective.

$$
(D v)_{(A,(\alpha, \beta), f, \theta)}(0, \cdot):\{0\} \oplus T_{(f, \theta)} \mathcal{P}_{2} \rightarrow T_{v(A,(\alpha, \beta), f, \theta)} \operatorname{Fred}_{n}\left(V_{1}, V_{2}\right) .
$$


proof. A proof here is a modification of that of [F, Prop. 4.9]. First we prove the following lemma:

Lemma 4.5. Assume that $(A,(\alpha, \beta)))$ is a solution to the Vafa-Witten equations (2.5) and (2.6) with $A$ irreducible and $(\alpha, \beta) \neq 0$ for some perturbation parameter $(f, \theta) \in \mathcal{P}_{2}$. If $b \in \Omega^{0,0}\left(X, \mathfrak{g}_{P}\right) \oplus \Omega^{0,2}\left(X, \mathfrak{g}_{P}\right)$ and $d \in \Omega^{0,1}\left(X, \mathfrak{g}_{P}\right)$ satisfy

$$
\left\langle D\left(\bar{\partial}_{A,(f, \theta)}+\bar{\partial}_{A,(f, \theta)}^{*}\right)_{(A,(f, \theta))}(\underline{f}, \underline{\theta}), d \otimes b^{*}\right\rangle_{L^{2}(X)}=0
$$

for all $(\underline{f}, \underline{\theta})$, then $d \otimes b^{*} \equiv 0$ on $X$.

proof. Suppose for a contradiction that $d \otimes b^{*} \neq 0$ on $X$. By varying $\underline{\theta}$, we see that $b$ and $d$ have orthogonal images in $\mathfrak{g}_{P}$ at each point $x \in X$ from Lemma 3.2. We then set $U:=\{b \neq 0\} \cap\{d \neq 0\} \subset X$. Then either $b$ or $d$ defines a subbundle $\xi_{1} \subset \mathfrak{g}_{P}$ on $U$ of $\operatorname{rank}_{\mathbb{R}}=2$. We define $\xi_{2}:=\left.\xi_{1}^{\perp} \subset \mathfrak{g}_{P}\right|_{U}$ so that $\left.\mathfrak{g}_{P}\right|_{U}=\xi_{1} \oplus \xi_{2}$. The connection $\left.A\right|_{U}$ on $\left.\mathfrak{g}_{P}\right|_{U}$ also splits into the following form:

$$
A=\left(\begin{array}{cc}
A_{1} & -\chi^{*} \\
\chi & A_{2}
\end{array}\right)
$$

where $A_{i}$ is a connection on $\xi_{i}$ for $i=1,2$, and $\chi \in \Omega^{1}\left(U, \xi_{2} \otimes \xi_{1}^{*}\right)$ is the second fundamental form. As $(A,(\alpha, \beta))$ is irreducible and non-zero section from the assumption, $\chi \neq 0$ on $U \subset X$. We suppose that $b \in \Omega^{0,0}\left(U, \xi_{1}\right) \oplus \Omega^{0,2}\left(U, \xi_{1}\right)$. We then get

$$
\begin{aligned}
D\left(\bar{\partial}_{A,(f, \theta)}+\bar{\partial}_{A,(f, \theta)}^{*}\right) & \\
=\sum_{i=1}^{4}\left(\underline{f}\left(v^{i}\right)\right) & \wedge \nabla_{A_{1}, v_{i}} b-\sum_{i=1}^{4} \iota(\underline{f}(\underline{f}, \underline{\theta}) b
\end{aligned}
$$

This turns out to be

$$
\left\langle\rho\left(\underline{f}_{x}\left(\chi_{x}\right)\right) b_{x}, d_{x}\right\rangle_{x}=0
$$

at each $x \in U$ and for all $\underline{f}_{x} \in \mathfrak{g r}\left(T^{*} X\right)_{x}$. Hence we get $d_{x} \otimes b_{x}^{*}=0$ at each $x \in U$ with $\chi_{x} \neq 0$. As $d_{x}^{-x} \otimes b_{x} \neq 0$ for all $x \in U$ from the assumption, we get $\chi=0$, thus, $\left.A\right|_{U}$ is reducible.

On the other hand, by a similar argument by Feehan-Lenes [FL, §5.3], one can obtain that, if $A$ is reducible on a non-empty open subset $U \subset X$ and $(\alpha, \beta) \neq 0, A$ is reducible on $X$. This is a contradiction. Therefore, $U \subset X$ is empty and $d \otimes b^{*} \equiv 0$ on $X$. 
We now suppose that $(D v)_{(A,(\alpha, \beta), f, \theta)}(0, \cdot)$ is not surjective. Then, there exist sections $b \in L_{k}^{2}\left(V_{1}\right)$ and $d \in L_{k-1}^{2}\left(V_{2}\right)$ with $d \otimes b^{*} \not \equiv 0$ on $X$ such that

$$
\left\langle D\left(\bar{\partial}_{A,(f, \theta)}+\bar{\partial}_{A,(f, \theta)}^{*}\right)_{(A,(f, \theta))}(\underline{f}, \underline{\theta}) b, d\right\rangle=0 .
$$

Then, from Lemma 4.5, we get $d \otimes b^{*} \equiv 0$. This is a contradiction. Therefore, $(D v)_{(A,(\alpha, \beta), f, \theta)}(0, \cdot)$ is surjective.

\subsection{No rank one and two loci}

In this section, we prove Proposition 1.4. As mentioned in the beginning of Section 4, once Propositions 4.2 and 4.4 are obtained, the proof of Proposition 1.4 goes along the same line with the case for the $P U(2)$-monopole equations $[\mathrm{F}, \S 4.6]$. Hence we give it sketchily.

First note that the map $s: \mathcal{C}^{*, 0}(P) \times \mathcal{P}_{2} \rightarrow L_{k-1}^{2}\left(\mathfrak{g}_{P} \otimes \Lambda^{0,1}\right) \times L_{k-1}^{2}\left(\mathfrak{g}_{P} \otimes\right.$ $\left.\left(\Lambda^{0,2} \oplus \Lambda^{1,1}\right)\right)$ is right semi-Fredholm, namely, the differential has closed range and finite dimensional cokernel. In particular,

$$
\mathbb{H}_{(A,(\alpha, \beta), p)}^{2}:=(\operatorname{Im}(D s(\cdot, p)))_{(A,(\alpha, \beta))}^{\perp}
$$

is a finite dimensional subspace of $L_{k-1}^{2}\left(\mathfrak{g}_{P} \otimes \Lambda^{0,1}\right) \times L_{k-1}^{2}\left(\mathfrak{g}_{P} \otimes\left(\Lambda^{0,2} \oplus \Lambda^{1,1}\right)\right)$. We denote by $\Pi_{(A,(\alpha, \beta))}$ the $L^{2}$-orthogonal projection from $L_{k-1}^{2}\left(\mathfrak{g}_{P} \otimes \Lambda^{0,1}\right) \times$ $L_{k-1}^{2}\left(\mathfrak{g}_{P} \otimes\left(\Lambda^{0,2} \oplus \Lambda^{1,1}\right)\right)$ to the $\operatorname{Im}(D s(\cdot, p))_{(A,(\alpha, \beta))}$.

Let $\left(c_{0}, p_{0}\right) \in M^{*, 0}(P)$. We consider the following composition:

$$
\Pi_{\left(c_{0}, p_{0}\right)} \circ s: \mathcal{B}^{*, 0}(P) \times \mathcal{P}_{2} \rightarrow\left(\mathbb{H}_{\left(c_{0}, p_{0}\right)}^{2}\right)^{\perp}
$$

Then the differential at $\left(c_{0}, p_{0}\right)$ of $\Pi_{\left(c_{0}, p_{0}\right)} \circ s$ is surjective, in particular, it is surjective on some open neighbourhood $\mathcal{U}_{\left(c_{0}, p_{0}\right)}$ of $\left(c_{0}, p_{0}\right)$ in $\mathcal{C}^{*, 0}(P) \times \mathcal{P}_{2}$. We set

$$
\mathcal{T}_{\left(c_{0}, p_{0}\right)}:=\mathcal{U}_{\left(c_{0}, p_{0}\right)} \cap\left(\Pi_{\left(c_{0}, p_{0}\right)} \circ s\right)^{-1}(0) \subset \mathcal{B}^{*, 0}(P) \times \mathcal{P}_{2}
$$

We denote by $\pi_{\mathcal{T}, \mathcal{P}_{2}}: \mathcal{T}_{\left(c_{0}, p_{0}\right)} \rightarrow \mathcal{P}_{2}$ the projection, and define $\left.\mathcal{T}_{\left(c_{0}, p_{0}\right)}\right|_{p}:=$ $\pi_{\mathcal{T}, \mathcal{P}_{2}}^{-1}(p) \cap \mathcal{T}_{\left(c_{0}, p_{0}\right)}$. We then prove the following:

Proposition 4.6. There is a first-category subset $\mathcal{P}_{2}^{\prime} \subset \mathcal{P}_{2}$, depending on $\left(c_{0}, p_{0}\right)$ such that for any $p \in \mathcal{P}_{2} \backslash \mathcal{P}_{2}^{\prime},\left.\mathcal{T}_{\left(c_{0}, p_{0}\right)}\right|_{p}$ contains no $(A,(\alpha, \beta), p)$ with $\alpha+\beta$ being of rank one nor two. 
proof. The argument consists of the following three steps: first, we consider the period map $v$ defined from $\mathcal{T}_{\left(c_{0}, p_{0}\right)}$ to $\operatorname{Fred}_{n}\left(V_{1}, V_{2}\right)$. As the differential of $v$ is not necessarily surjective, we stabilize the map to obtain a submersion $v^{\prime}: \mathcal{V}_{\left(c_{0}, p_{0}\right)} \times \mathcal{T}_{\left(c_{0}, p_{0}\right)} \rightarrow \operatorname{Fred}_{n}\left(V_{1}, V_{2}\right)$, where $\mathcal{V}_{\left(c_{0}, p_{0}\right)}$ is some finite dimensional vector space in $T_{v\left(c_{0}, p_{0}\right)} \operatorname{Fred}_{n}\left(V_{1}, V_{2}\right)$. Second, we lift the stabilized period map $v^{\prime}$ to $\mathcal{V}_{\left(c_{0}, p_{0}\right)} \times \mathcal{T}_{\left(c_{0}, p_{0}\right)} \rightarrow \operatorname{Flag}_{k, n}\left(V_{1}, V_{2}\right)$ as the rank one and two loci $\tilde{J}_{k}\left(Z^{\prime}\right)$ lives in $\operatorname{Flag}_{k, n}\left(V_{1}, V_{2}\right)$. This is again not necessarily a submersion, so we stabilize it to obtain a smooth submersion $w^{\prime}: \mathbb{C}^{k} \times \mathcal{W}_{\left(c_{0}, p_{0}\right), k} \rightarrow \operatorname{Flag}_{k, n}\left(V_{1}, V_{2}\right)$, where $\mathcal{W}_{\left(c_{0}, p_{0}\right), k}$ is a submanifold of $\mathcal{V}_{\left(c_{0}, p_{0}\right)} \times \mathcal{T}_{c_{0}, p_{0}}$ with finite codimension. Third, we use the Sard-Smale theorem (Proposition 4.1) to the $w^{\prime}$ to obtain the assertion.

Step 1. First, we consider the period map $v: \mathcal{T}_{\left(c_{0}, p_{0}\right)} \rightarrow \operatorname{Fred}_{n}\left(V_{1}, V_{2}\right)$. From Proposition 4.4, the operator

$$
(D v)_{\left(c_{0}, p_{0}\right)}:\{0\} \oplus T_{p_{0}} \mathcal{P}_{2} \rightarrow T_{v\left(c_{0}, p_{0}\right)} \operatorname{Fred}_{n}\left(V_{1}, V_{2}\right)
$$

is surjective. On the other hand, we have

$$
T_{\left(c_{0}, p_{0}\right)} \mathcal{T}_{\left(c_{0}, p_{0}\right)}+\left(\{0\} \oplus T_{p_{0}} \mathcal{P}_{2}\right)=\mathbb{H}_{\left(c_{0}, p_{0}\right)}^{1} \oplus T_{p_{0}} \mathcal{P}_{2},
$$

where

$$
\begin{aligned}
\mathbb{H}_{\left(c_{0}, p_{0}\right)}^{1} & :=\operatorname{ker}\left(D s\left(\cdot, p_{0}\right)\right)_{\left(c_{0}, p_{0}\right)} \\
& =\operatorname{ker}\left(\Pi_{\left(c_{0}, p_{0}\right)} \circ(D s)\left(\cdot, p_{0}\right)\right)_{\left(c_{0}, p_{0}\right)} \\
& =\operatorname{ker}\left(D \pi_{\mathcal{T}, \mathcal{P}_{2}}\right)_{\left(c_{0}, p_{0}\right)} \subset T_{\left(c_{0}, p_{0}\right)} \mathcal{C}^{*, 0}(P) .
\end{aligned}
$$

Hence, $(D v)_{\left(c_{0}, p_{0}\right)}: \mathbb{H}_{\left(c_{0}, p_{0}\right)}^{1} \oplus T_{p_{0}} \mathcal{P}_{2} \rightarrow T_{v\left(c_{0}, p_{0}\right)} \operatorname{Fred}_{n}\left(V_{1}, V_{2}\right)$ is surjective.

As [F, Lem 4.15], we also have the following isomorphism.

$$
\left(\mathbb{H}_{\left(c_{0}, p_{0}\right)}^{1} \oplus T_{p_{0}} \mathcal{P}_{2}\right) \cong T_{\left(c_{0}, p_{0}\right)} \mathcal{T}_{\left(c_{0}, p_{0}\right)} \oplus \operatorname{coker}\left(D \pi_{\mathcal{T}, \mathcal{P}_{2}}\right)_{\left(c_{0}, p_{0}\right)}
$$

We then define the following finite dimensional vector space.

$$
V_{\left(c_{0}, p_{0}\right)}:=(D v)_{\left(c_{0}, p_{0}\right)}\left(\operatorname{coker}\left(D \pi_{\mathcal{T}, \mathcal{P}_{2}}\right)_{\left(c_{0}, p_{0}\right)}\right) \subset T_{v\left(c_{0}, p_{0}\right)} \operatorname{Fred}_{n}\left(V_{1}, V_{2}\right) .
$$

We denote the inclusion by $i: V_{\left(c_{0}, p_{0}\right)} \rightarrow T_{v\left(c_{0}, p_{0}\right)} \operatorname{Fred}_{n}\left(V_{1}, V_{2}\right)$. We then define

$$
v^{\prime}: V_{\left(c_{0}, p_{0}\right)} \times \mathcal{T}_{\left(c_{0}, p_{0}\right)} \rightarrow \operatorname{Fred}_{n}\left(V_{1}, V_{2}\right)
$$

by $v^{\prime}(y,(c, p)):=i(y)+v(c, p)$ for $(y,(c, p)) \in V_{\left(c_{0}, p_{0}\right)} \times \mathcal{T}_{\left(c_{0}, p_{0}\right)}$. As the differential of $v^{\prime}$ is surjective at $\left(0, c_{0}, p_{0}\right)$, there exists an open neighbourhood of the origin $\mathcal{V}_{\left(c_{0}, p_{0}\right)} \subset V_{\left(c_{0}, p_{0}\right)}$ such that the restriction

$$
v^{\prime}: \mathcal{V}_{\left(c_{0}, p_{0}\right)} \times \mathcal{T}_{\left(c_{0}, p_{0}\right)} \rightarrow \operatorname{Fred}_{n}\left(V_{1}, V_{2}\right)
$$


is a submersion.

We now consider the following for $k \geq n$.

$$
\mathcal{W}_{\left(c_{0}, p_{0}\right), k}:=\left(\mathcal{V}_{\left(c_{0}, p_{0}\right)} \times \mathcal{T}_{\left(c_{0}, p_{0}\right)}\right) \cap\left(v^{\prime}\right)^{-1}\left(\operatorname{Fred}_{k, n}\left(V_{1}, V_{2}\right)\right)
$$

As (4.1) is a submersion, the above $\mathcal{W}_{\left(c_{0}, p_{0}\right), k}$ is a smooth submanifold with finite codimension in $\mathcal{V}_{\left(c_{0}, p_{0}\right)} \times \mathcal{T}_{\left(c_{0}, p_{0}\right)}$, thus, $\mathcal{V}_{\left(c_{0}, p_{0}\right)} \times \mathcal{T}_{\left(c_{0}, p_{0}\right)}=\bigcup_{k \geq n} \mathcal{W}_{\left(c_{0}, p_{0}\right), k}$ is a countable disjoint union of smooth manifolds.

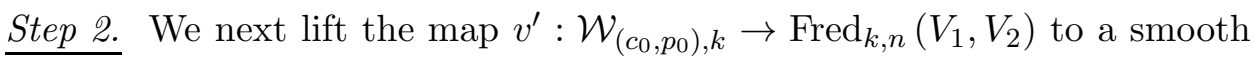
map

$$
w: \mathcal{W}_{\left(c_{0}, p_{0}\right), k} \rightarrow \operatorname{Flag}_{k, n}\left(V_{1}, V_{2}\right)
$$

by $(y,(A,(\alpha, \beta)), p) \mapsto([(\alpha, \beta)], i(y)+v((A,(\alpha, \beta)), p)$. This is again not necessarily a submersion, so we stabilize it as described below.

Let $\left(y_{1},\left(c_{1}, p_{1}\right)\right)$ in $\mathcal{W}_{\left(c_{0}, p_{0}\right), k}$. Since a countable union of first category subsets is a first category subset and $\mathcal{W}_{\left(c_{0}, p_{0}\right), k}$ is paracompact, we only consider a single open neighbourhood of $\left(y_{1},\left(c_{1}, p_{1}\right)\right)$.

We take an orthonormal basis $\left\{b_{1, j}\right\}_{j=1}^{k}$ of the kernel of $v^{\prime}\left(y_{1},\left(c_{1}, p_{1}\right)\right)=$ $i\left(y_{1}\right)+v\left(c_{1}, p_{1}\right)$. We denote by

$$
\pi_{(y,(c, p))}: L_{k-1}^{2}\left(\mathfrak{g}_{P} \otimes\left(\Lambda^{0,0} \oplus \Lambda^{0,2}\right)\right) \rightarrow \operatorname{ker}(i(y)+v(c, p))
$$

the smooth family of $L^{2}$-orthogonal projection. We then consider a smooth map

$$
w^{\prime}: \mathbb{C}^{k} \times \mathcal{W}_{\left(c_{0}, p_{0}\right), k} \rightarrow \operatorname{Flag}_{k, n}\left(V_{1}, V_{2}\right)
$$

defined by

$$
w^{\prime}(z, y, c, p) \mapsto\left(\left[(\alpha, \beta)+\pi_{(y,(c, p))}\left(\sum_{j=1}^{k} z_{j} b_{1, j}\right)\right], i(y)+v(c, p)\right)
$$

where $z=\left(z_{1}, \ldots, z_{k}\right) \in \mathbb{C}^{k}$. As [F, Claim 4.18], the map $w^{\prime}$ is a submersion at $\left(0, y_{1},\left(c_{1}, p_{1}\right)\right)$, thus $\mathcal{W}_{\left(c_{0}, p_{0}\right), k}^{\prime}:=\left(w^{\prime}\right)^{-1}\left(\tilde{J}_{k}\left(Z^{\prime}\right)\right)$ is a $C^{\infty}$-Banach submanifold of $\mathbb{C}^{k} \times \mathcal{W}_{\left(c_{0}, p_{0}\right), k}$.

Step 3. We are now in a situation to invoke the Sard-Smale theorem (Prop.

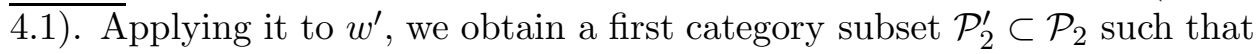
for $p \in \mathcal{P}_{2} \backslash \mathcal{P}_{2}^{\prime}$

$\operatorname{codim}_{\mathbb{R}}\left(\left.\mathcal{W}_{\left(c_{0}, p_{0}\right), k}^{\prime}\right|_{p}, \mathbb{C}^{k} \times\left.\mathcal{W}_{\left(c_{0}, p_{0}\right), k}\right|_{p}\right)=\operatorname{codim}_{\mathbb{R}}\left(\tilde{J}_{k}\left(\mathcal{Z}^{\prime}\right), \operatorname{Flag}_{k, n}\left(V_{1}, V_{2}\right)\right)$ 
Since $\operatorname{codim}_{\mathbb{R}}\left(\tilde{J}_{k}\left(\mathcal{Z}^{\prime}\right), \operatorname{Flag}_{k, n}\left(V_{1}, V_{2}\right)\right)=\infty$ but $\operatorname{dim}_{\mathbb{R}}\left(\mathbb{C}^{k} \times\left.\mathcal{W}_{\left(c_{0}, p_{0}\right), k}\right|_{p}\right)<$ $\infty$, we deduce that $\left.\mathcal{W}_{\left(c_{0}, p_{0}\right), k}^{\prime}\right|_{p}$ is empty.

We also have $\left.\left.\left.\mathcal{T}_{\left(c_{0}, p_{0}\right)}\right|_{p} \cap w\right|_{\mathcal{T}_{\left(c_{0}, p_{0}\right)}}(\cdot, p)^{-1}\left(\tilde{J}_{k}\left(\mathcal{Z}^{\prime}\right)\right) \subset \mathcal{W}_{\left(c_{0}, p_{0}\right), k}^{\prime}\right|_{p}$. Since $\left.\mathcal{W}_{\left(c_{0}, p_{0}\right), k}^{\prime}\right|_{p}$ is empty, thus so is $\left.\left.\mathcal{T}_{\left(c_{0}, p_{0}\right)}\right|_{p} \cap w\right|_{\mathcal{T}_{\left(c_{0}, p_{0}\right)}}(\cdot, p)^{-1}\left(\tilde{J}_{k}\left(\mathcal{Z}^{\prime}\right)\right)$. Hence $\mathcal{T}_{\left(c_{0}, p_{0}\right)}$ has no rank one or two section $\alpha+\beta$ for $\operatorname{dim} \operatorname{ker}\left(\bar{\partial}_{A, p}+\bar{\partial}_{A, p}^{*}\right)=k$ and $p \in \mathcal{P}_{2} \backslash \mathcal{P}_{2}^{\prime}$. Since a countable union of first category subsets is a first category subset, we get the assertion by repeating this for $k \geq n$.

Proof of Proposition 1.4. By Proposition 4.6, $M^{*, 0}(P) \cap \mathcal{T}_{\left(c_{0}, p_{0}\right)} \subset \mathcal{T}_{\left(c_{0}, p_{0}\right)}$ has no rank one nor two solution $(A,(\alpha, \beta), p)$ for $p \in \mathcal{P}_{2} \backslash \mathcal{P}_{2}^{\prime}$. By repeating this argument for each $(A,(\alpha, \beta), p) \in \mathcal{C}^{*, 0}(P) \times \mathcal{P}_{2}$, we obtain a first category subset for each open neighbourhood of it. As $\mathcal{C}^{*, 0}(P) \times \mathcal{P}_{2}$ is paracompact, we can cover $M^{*, 0}(P)$ by countable such open neighbourhoods. Since a countable union of first category subsets of $\mathcal{P}_{2}$ is again a first category subset of $\mathcal{P}_{2}$, we get the assertion.

\section{References}

[DK] S. K. Donaldson and P. B. Kronheimer, The Geometry of FourManifolds, Oxford University Press, New York, 1990.

[F] P. M. N. Feehan, Generic metrics, irreducible rank-one PU(2) monopoles, and transversality, Comm. Anal. Geom. 8 (2000), 905-967.

[FL] P. M. N. Feehan and T. G. Leness, PU(2) monopoles. I : Regularity, Uhlenbeck compactness, and transversality, J. Differential Geom. 49 (1998), 265-410.

[Ha] A. Haydys, Fukaya-Seidel category and gauge theory, J. Symplectic Geom. 13 (2015), 151-207.

[LM] H. B. Lawson and M-L. Michaelshon, Spin geometry, Princeton University Press, Princeton, NJ, 1989.

[Ma] B. Mares, Some Analytic Aspects of Vafa-Witten Twisted $\mathcal{N}=4$ Supersymmetric Yang-Mills theory, Ph.D thesis, M.I.T., 2010.

[Mo] J. W. Morgan, The Seiberg-Witten equations and application to the topology of smooth four-manifolds, Princeton Univ. Press, Princeton, NJ, 1996. 
[Ta] Y. Tanaka, Some boundedness property of the Vafa-Witten equations on closed four-manifolds, Q. J. Math. 68 (2017), 12031225 .

[Te] A. Teleman, Moduli spaces of $P U(2)$-monopoles, Asian J. Math. 4 (2000), 391-435.

[VW] C. Vafa and E. Witten, A strong coupling test of S-duality, Nucl. Phys. B, 432, (1994), 484-550.

[W] E. Witten, Fivebranes and Knots, Quantum Topol. 3 (2012), $1-137$.

Mathematical Institute, University of Oxford

Radcliffe Observatory Quarter, Woodstock Road, Oxford, OX2 6GG, U.K. tanaka@maths.ox.ac.uk 